\title{
Study on the Scientific Research Group Management of Chinese High Technology Enterprises from the Perspective of Knowledge Transfer
}

\author{
Wan Ting ${ }^{\text {a,* }}$ \\ ${ }^{a}$ Zhejiang Jinji Property Corporation Limited, Hangzhou, China
}

\begin{abstract}
Knowledge transfer is the foundation of group scientific and research activities, and the life line of the knowledge innovation of science and research group. However, there are various difficulties for the internal knowledge transfer in the science and research group of high technology enterprises, which hinders the improvement of the knowledge innovation and performance of the science and research groups of high technology enterprises. The knowledge transfer situation in the science and research groups of high technology enterprises is worrying. The key of the management of the science and research group in high technology enterprises is the cooperation among members. For the group operation, only mutual trust is not sufficient. There should be effective encouraging and restriction mechanism to motivate members' enthusiasm of knowledge transfer and avoid members' moral risks.
\end{abstract}

Index Terms: Science and Research Group; High Technology Enterprise; Transfer Knowledge; National Innovation System; Encouraging and Restriction Mechanism

(C) 2012 Published by MECS Publisher. Selection and/or peer review under responsibility of the International Conference on E-Business System and Education Technology

\section{Introduction}

High technology enterprises are an important part of national innovation system with the task to cultivate talents for the social and economic development, and increase the social knowledge stock. High technology enterprises are the main force of the construction of innovative country. With more and more knowledge involving in the science and research projects and the enlargement of the science and research scale, the knowledge innovation more and more depends on the group work. Therefore, the knowledge transfer efficiency among the science and research group members directly influences the knowledge productivity of the group. How to promote the knowledge transfer in the group scientific and research activities and improve the knowledge transfer efficiency should be an important issue in the construction and management of science and research group in high technology enterprises.

* Corresponding author:

E-mail address: * yanjiulilun2@126.com 


\section{Knowledge Transfer Is the Foundation of Knowledge Innovation}

The science and research group uses knowledge as the basic material to provide the society with knowledge or knowledge based services. Knowledge leading and knowledge innovation are the key to construct the competitive advantage of science and research group, which is also the symbol of the scientific and research strength of the high technology enterprises. In the knowledge production chain of the science and research group, knowledge transfer is the most basic and critical link. It means the process of the transfer of knowledge among individuals in different ways, which is the life line of knowledge innovation in science and research group and the foundation to improve the knowledge productivity. Although not all of the scientific and research achievements come from group work, single and individual work is more and more difficult to meet the requirements of modern great science, great projects. In the information explosive era, it is difficult for individuals to accurately obtain the required effective information in time in the large knowledge ocean. However, the full knowledge communication and sharing among members of science and research group can effectively make up the restriction of individuals, which may optimize individual knowledge structure and increase individual knowledge stock. In the meantime, the transfer of knowledge among group members with different knowledge structure and depth may enable the group members to have deeper thoughts, broader thinking and more research methods in the knowledge sharing and thoughts collision to form fuller research perspective. The shock and enlargement effect of the knowledge transfer effectively promotes the innovative thinking. Therefore, for great projects and great science, group scientific and research work have the advantages which individual work does not have. The internal knowledge transfer of the group is one of the main characteristics of group science and research, and has penetrated into every link of the group scientific and research activities. From the perspective of knowledge flow, the group internal knowledge transfer has two directions, i.e. the vertical knowledge transfer from the group leaders to members, and the horizontal knowledge transfer among group members. The leader of the science and research group is the general designer and engineer who leads the research direction, is responsible to divide the research target and assign the research task. He passes the academic thoughts and scientific and research originality to group members, and the group members conduct research work on basis of it to transform it into specific scientific and research achievements. In the vertical knowledge transfer, the group leaders guide members to transfer knowledge and thoughts. In the meantime, members also influence the science and research originality of the leaders. The vertical knowledge transfer includes the knowledge transfer among members in different levels. The horizontal knowledge transfer refers to the transfer of knowledge among members with minor differences in scientific and research accumulation and experience. Both vertical knowledge transfer and horizontal knowledge transfer greatly influence the group cooperation atmosphere and innovation thoughts, which is the main factor of knowledge innovation and group performance.

\section{Main Difficulties in the Knowledge Transfer of the Science and Research Group of High Technology Enterprises}

\subsection{Bad academic atmosphere damages the enthusiasm of knowledge transfer}

Knowledge is the most important resource of the scientific and research workers. Knowledge is nonexclusive and can be copied, which provides the foundation for knowledge sharing and transfer while the knowledge sharing and transfer may accelerate the knowledge innovations to promote the knowledge transform into productivity. However, when the academic atmosphere is not good, such characteristics of knowledge may open the door for some opportunist, causing many infringements and academic corruption. In the science and research groups of high technology enterprises, there is also plagiarism. For the researchers, the academic thoughts and scientific and research originality is the soul of science and research. The research process is the 
process to prove the scientific and research originality. The scientific and research achievement is the explicit knowledge transferred by tacit knowledge such as the scientific and research originality. The improper academic atmosphere of sharing the scientific and research originality and thought while ignoring the value of the originality providers seriously damages the enthusiasm of members to transfer knowledge.

\subsection{Not willing to transfer knowledge}

There is a potential knowledge market inside the organization, and there are knowledge transactions between sellers and buyers. According to the decision theory of Simon, the owners of knowledge firstly make the plan: transfer knowledge or do not transfer knowledge, and then make evaluation to choose the plan which can bring more benefits. The selected plan should satisfy two conditions, i.e. the benefit is more than the cost; the remaining benefits of one plan is higher than that of the other one. Under the following two situations, the owners of knowledge will choose not to transfer the knowledge: first, if the knowledge transfer cannot bring relevant value corresponding to the contribution, there might be insufficient motivation. When choosing the knowledge transfer plan, the owners of knowledge may expect to have more scientific and research achievements through cooperation besides the emotional experience such as reputation, prestige, thus obtaining higher academic reputation, more scientific and research expenditure and better career prospect. However, the benefits of the knowledge transfer are uncertain. Therefore, the knowledge transfer plan will not be selected if the expected benefits are not high. When the actual benefits of the knowledge transfer are less than the expected, the knowledge transfer can not be maintained. Second, if the non-transfer plan can bring more benefits to the owners of knowledge, there will be insufficient will to transfer. If the knowledge is the achievement of specific research through hard work, the ownership of knowledge indicates the prestige and position of the owner in certain area. When the knowledge owners consider that the monopoly interests of the knowledge are higher than the loss of not transferring the knowledge, he will choose not to transfer.

\subsection{Not willing to accept the knowledge}

The will of the recipient to accept the knowledge has a significant influence on the knowledge transfer performance. If there is no acceptance will, it will be difficult for the knowledge transfer to succeed. Just like passive study, if the knowledge recipient is reluctant to receive knowledge, he/she will have repulsion in mind. There are many situations in high technology enterprises that members do not want to accept knowledge, the reasons of which are mainly as follows: first, insufficient time and energy. Most of the science and research group members are scientific and research personal of the high technology enterprises. Besides science and research task, they have other burdensome tasks in management, which are relatively complicated. As such, many members do not have enough time and energy to participate in the knowledge transfer activities. Second, there is no expectation about the group internal knowledge transfer. Some researchers consider that they have grasped rich knowledge, and other members may not have more novel and valuable knowledge. Therefore, they do not have high expectations on receiving the knowledge transfer, and are reluctant to participate in the knowledge transfer. Third, there is a problem of "face", so they do not want to modestly receive the knowledge transfer. Among the scientific and research personal in high technology enterprises, there are a few who love "face" very much and do not want to make a request for knowledge worrying about that people may consider them narrow and superficial in knowledge. Instead, they set certain barriers for receiving the knowledge transfer. Fourth, some science and research groups do not have clear research direction, causing that members do not have clear request for knowledge, such as certain groups at the initial stage. They do not have clear project guidance and are in the process of exploring. Correspondingly, their members do not have sufficient receiving will without clear request for knowledge. 


\subsection{The knowledge transfer path is not properly selected, causing difficulties of knowledge transfer}

The knowledge owned by people can be divided as explicit knowledge and tacit knowledge. The former is relatively objective and can be clearly expressed by words, numbers and symbols, while the latter is relatively subjective, closely related to situations and experience of the knowledge owners, which can not be expressed, formalized and filed clearly by words. What one knows is much more than what he can explains, i.e. the tacit knowledge people have is much more than the explicit knowledge they have. Because of different characteristics, explicit knowledge and tacit knowledge have different knowledge transfer paths. For explicit knowledge, it is proper to adopt the word transfer path which helps maintain information and knowledge spread. For tacit knowledge, it is proper to use face to face communication, which can have feedback in time and can make repeated communication to improve the receiving effect. However, in reality, members of domestic science and research groups do not often use face to face communication to transfer knowledge, and the face to face communication among members is mainly used to communicate daily matters while the knowledge transfer depends on internet and communication tools. As such, improper transfer path increases the difficulties of effective knowledge transfer among science and research group members.

\section{The Management of The Science and Research Groups of High Technology Enterprises on Basis of Knowledge Transfer}

\subsection{Cultivate the knowledge sharing culture in science and research groups}

The culture of the science and research group, i.e. the literal characteristic of the group, is the attitude and habitual reaction of members towards things. Because of the great influence, it becomes the common behavior of members and impacts the attitude and behavior selection of the members. The knowledge transfer performance of the group is closely related to whether there is a sharing culture. The reason for a team to become group is that there is interaction and sharing among members, which makes the whole performance of the group much better the total of personal performance. Therefore, the group without a sharing culture is a fake group. Without cultural support, the knowledge sharing mechanism can not produce the expected effect however mature it is. The establishment and implementation of sharing mechanism can effectively promote the generation of a sharing culture. However, the process for the establishment of the mechanism to the formation of the culture is long, which can be divided as several psychological stages such as members' recognition of the sharing system, achievement of the sharing expectation, formation of the sharing value, habitual maintaining of the sharing activities before the knowledge sharing culture can become the dominating culture of the group. Therefore, in the original science and research group lacking sharing culture, the establishment of sharing mechanism can not rapidly improve the knowledge transfer performance at the beginning, but at the end it will. The establishment of the sharing mechanism in science and research groups should have the following three conditions. First, the science and research group should have leaders with good sentiment who advocates science and knowledge, enjoy the process of pursuing knowledge and truth, and are ready to guide juniors and students. Although they cannot be as god to abandon reputation and interests, they would neither put reputation and interests before academy, nor put individual interests before junior's interests. They make their academic prestige by deep foundation, rich research experience and advance perspective, and impel the whole group by their personality. They are not necessarily the administrative leaders at the same time, but they are the actual spiritual leaders of the group, leading the group proceeding with the knowledge sharing. Second, build good sharing system. The style of the great scholar is the spiritual guide for the group to form sharing culture while standardization of the system design is the basis of it. Members could not have clear expectations on the results of the sharing activities only with the spiritual guide, while system may be helpful in this respect. System design combined with the model effect of leaders will surely enhance the confidence and will of the members 
to share knowledge. Third, respect the academic freedom. Scholars are persons having creative activities. They are not blind to authority and restricted to fixed forms. Instead, they have active thoughts and expect their views can be respected. Therefore, the science and research group in high technology enterprises should form the active cultural atmosphere will academic freedom, encourage members to have objective, diligent thinking and make active discussions to fully enjoy the knowledge sharing.

\subsection{Establish multiple models of knowledge transfer}

The internal knowledge transfer in the science and research groups of high technology enterprises can use the face to face communication path, telephone path, email path, documentation path, etc. Different paths are suitable for transferring different types of information. Tacit knowledge can not be easily coded, and is ambiguous. The transfer often needs many methods such as metaphor, analogy, paradigm, inducement, simulation, etc. Face to face path is the best choice to transfer tacit knowledge because it is convenient for knowledge senders to choose additional methods in the course of transfer according to situations, for example gestures, facial expressions, even writing, drawing, etc to make the tacit knowledge explicit. In the meantime, face to face communication can have in time feedback, which helps the knowledge senders effectively code information. The explicit knowledge can be easily coded and clearly expressed. Therefore, the transfer of explicit knowledge can use Email, QQ, MSN, files which are more effective. The word path is good for the maintaining of information, and may effectively reduce the negative influence caused by forgetting. The recipients can also have a deep understanding. Because the tacit knowledge members own is much more than the explicit knowledge, the science and research group should build multiple paths centering on face to face communications and supplemented by telephone, Email, QQ, MSN, files, etc. The face to face communication mechanism in science and research groups should by systemized with regular report meetings, discussions to strengthen individual and group study and promote the transfer of tacit knowledge. In the meantime, the science and research groups should encourage members to have non-formal communications through the above paths, which can not only promote the emotional communication, but also promote the knowledge transfer.

\subsection{Improve the protection mechanism of the group internal knowledge transfer}

Like the intellectual property system, the science and research groups also need to improve the knowledge protection mechanism to safeguard the interests of knowledge providers. Currently, knowledge reporting system and knowledge checking system is the method commonly used in the knowledge management in enterprises. Knowledge reporting system refers to that employees regularly report their knowledge contribution to seniors, while knowledge checking system means that leaders or persons responsible for knowledge management evaluate the truth and effectiveness of what employees report to identify employees' contributions and improve their enthusiasm of knowledge transfer. However, neither knowledge reporting system nor knowledge checking system can define the contribution of knowledge transfer before innovative achievements are achieved. It is necessary to improve reports in respect of the science and research originality, the person who put forward the originality, tutors, specific researchers to prove the originality, etc to identify employees' contributions and provide basis for the reporting of knowledge innovation achievements and sharing. The group can vote the knowledge checking team to review the innovative achievement reporting of members and provide objective evaluation. This can not only helps recognize the contributors' value, but also can avoid "free ride". If the group internal knowledge transfer protection mechanism can be improved, there will be less request of knowledge monopoly, which can in turn increase the willing of knowledge transfer. 


\subsection{Complete the encouraging mechanism of knowledge transfer}

The research finds out that encouragement can promote the owners of knowledge to transfer knowledge. However, incomplete knowledge transfer encouragement mechanism only can increase the knowledge sharing, but can not significantly increase the quantity and quality of individual transfer of knowledge. In order to avoid fake situations of hardworking of employees, the science and research group should build the knowledge transfer encouragement mechanism on basis of individual performance. The performance evaluation of the knowledge transfer will not automatically check the quantity of the participation in knowledge sharing. Instead, it combines employees' attitude in the activities and the contributions, etc. The evaluation of knowledge contribution may refer to the results of knowledge reporting and knowledge checking. The group should give awards to the employees with good performance. The material rewards include money bonus, science and research budget, etc. There are more spiritual rewards, such as recognition, reputation, promotion, signature, etc. Some rewards are of both material and spiritual meaning, such as taking part in international meetings, study opportunities, etc. It should be mentioned that according to the expectation theory, different encouragement methods have different attractions. It is necessary to choose proper method on basis of individual requirement. Punishment and reward are two aspects of the encouragement mechanism. The punishment mechanism restricts the improper activities of the knowledge transfer sharing, criticize the infringement, expropriate the illegal benefits, compulsory quit, etc to protect the enthusiasm of the knowledge owners to transfer knowledge.

\section{Conclusion}

As the basic science and research organization, the science and research group in high technology enterprises plays an important role in national innovation system. Knowledge transfer is a main characteristic of group science and research activities, and the basic link of knowledge innovations. The performance of the group internal knowledge transfer directly influences the group ability of knowledge innovation. The science and research group members of high technology enterprises have different backgrounds, skills and knowledge. Their knowledge can supplement each other, which determines the mutual dependence of the group members. The cooperation among members determines the achievement of the group target. Therefore, the key of the management of the science and research groups in high technology enterprises is the cooperation among members. The group operation can not only depend on the mutual trust. It is also necessary to build effective encouragement and restriction mechanism to motivate members' enthusiasm to transfer knowledge and avoid their moral risks.

\section{Acknowledgements}

This thesis was supported by the Construct Program of the Key Discipline of Shanghai Municipal Education Commission (No.J51702), Philosophy and Society Science Foundation of Zhejiang Province (No. 10CGGL01ZQ) and Natural Science Foundation of Zhejiang Province (No. Y6080012)

\section{References}

[1] Zhou Haiyan, "On the Path Selection to Upgrade the Competitiveness of Chinese Textile Industry in the Post-quota Era,"Advances in Management of Technology (Proceedings of the International Conference on Management of Technology(Part 1), Marrickville: Aussino Academic Publishing House Press ,2008,pp.776-779 
[2] ZHOU Haiyan, "On the Improvement of the Independent Innovation Ability of Chinese Manufacture Industry by the Technological Spillover Effect of FDI,'Proceedings of 2009 International Conference on Management Science and Engineering, Marrickville: Orient Academic Form Press,2009,pp. 26-29

[3] Hao Jiao, Jiang Wei, and Yu Cui, "An empirical study on paths to develop dynamic capabilities: From the perspectives of entrepreneurial orientation and organizational learning. Frontiers of Business Research in China. 2010(1), pp. 47-72.

[4] ZHOU Haiyan and CHENG Xinzhang,"On the Constitution of Risk Management System of Chinese Enterprises Overseas M\&As, "Proceedings of 2008 Conference Regional Economy and Sustainable Eevelopment, Allawah:M\&D Forum Press,2008, pp. 563-566 